\title{
Treatment of TRIC infection of the eye with rifampicin or chloramphenicol
}

\author{
S. DAROUGAR, M. VISWALINGAM, J. D. TREHARNE, J. R. KINNISON, \\ AND B. R. JONES \\ From the WHO Collaborating Centre for reference and research on trachoma and other chlamydial infections; \\ and Virus Laboratory, Department of Clinical Ophthalmology, Institute of Ophthalmology, \\ London WC1H $9 Q S$
}

SUMMARY An open trial was carried out on 63 patients in London to assess the efficacy of $1 \%$ rifampicin eye ointment in comparison with $1 \%$ chloramphenicol eye ointment in the treatment of sexually transmitted TRIC infection of the eye. Patients included were selected on the basis of positive cultures for Chlamydia trachomatis.

Three weeks' treatment with rifampicin eye ointment used 3 times daily was not sufficient to cure the disease, but a 6 or 7 week course gave $90 \%$ clinical and microbiological cure rate. Treatment with chloramphenicol eye ointment 3 times daily for 4 to 6 weeks failed to cure the disease.

Rifampicin, a semisynthetic antibiotic, irreversibly inhibits the growth of $C$. trachomatis (i.e., TRIC agents and lymphogranuloma venereum [LGV] agents) apparently by the inhibition of the agent's DNA-dependent RNA polymerase (Becker, 1972; Becker et al., 1970).

A clinical trial in schoolchildren with active hyperendemic trachoma in Southern Tunisia (Dawson et al., 1975a) showed that rifampicin eye ointment twice daily for 10 weeks was substantially more beneficial than boric acid ointment, but it appeared to have no advantages over tetracycline eye ointment.

Several authors have claimed that chloramphenicol topical therapy is effective against trachoma (Choumakov et al., 1951; Leo, 1951; Scuderi and Bellomio, 1952; Vasilieva, 1958). However, we have repeatedly isolated $C$. trachomatis from the eyes of persons with continuing active disease despite treatment with chloramphenicol eye drops or ointment before referral to us.

The purpose of this study was to assess in a carefully monitored open trial, using various regimens of treatment, the efficacy of rifampicin eye ointment in comparison with chloramphenicol eye ointment in the treatment of paratrachoma, i.e., sexually transmitted TRIC infection of the eye.

\section{Subjects and methods}

SELECTION OF PATIENTS

The patients included in this study were selected

Address for reprints: Dr S. Darougar, Institute of Ophthalmology, Judd Street, London WC1H 9QS. on the basis of positive cultures for TRIC agent from patients with acute follicular conjunctivitis referred to the External Eye Disease Clinic at Moorfields Eye Hospital. Patients who had symptoms of concurrent genital infection were excluded from this study and were referred to the diagnostic clinic for investigation and treatment with oral tetracycline, as were those who were not able to attend for 12 weeks' follow-up.

\section{CLINICAL EXAMINATION}

Clinical signs observed with a Haag-Streit 900 slitlamp and symptoms were recorded on a proforma using an extension of the scoring system used for the study of trachoma (Dawson et al., 1975b). The symptoms and signs were graded on a 0 to 3 scale, as indicated in Table 1 . The highest possible score was 92: 12 for symptoms and 80 for signs in any one eye.

\section{LABORATORY INVESTIGATIONS}

Conjunctival swabbings collected from different areas of the conjunctiva were placed in plastic capsules containing 2SPS transport medium (Darougar and Jones, 1971) and stored in a liquid nitrogen refrigerator until transported to the laboratory, where they were stored in a refrigerator at $-70^{\circ} \mathrm{C}$ until the time of inoculation. The methods of culture in irradiated McCoy cells and the identification of isolates have been described elsewhere (Darougar et al., 1970), also the method of measuring the antibiotic sensitivity of chlamydial isolates in cell culture (Treharne et al., 1977). 
Table 1 The maximum scoring value of symptoms and signs observed in TRIC infection of the eye

\begin{tabular}{ll}
\hline Symptoms & Maximum score \\
Lachrymation & 3 \\
Discharge & 3 \\
Grittiness & 3 \\
Photophobia & 3 \\
Signs & \\
Bulbar conjunctiva & \\
Hyperaemia & 3 \\
Limbus & \\
Oedema & 3 \\
Pannus & 5 \\
Cornea & 3 \\
Epithelial punctate keratitis & 3 \\
Subepithelial punctate keratitis & 3 \\
$\begin{array}{l}\text { Palpebral conjunctiva } \\
\text { (Scores for each of 3 areas of }\end{array}$ & \\
$\quad$ conjunctiva, i.e., upper tarsus, \\
$\quad$ upper fornix, and lower lid, were \\
$\quad$ recorded separately) & 3 \\
Hyperaemia & 3 \\
Diffuse infiltration & 3 \\
Papillae & 3 \\
Follicles-area & 3 \\
Follicles-size & 3 \\
Scar & 3
\end{tabular}

\section{MEDICATION}

Either $1 \%$ rifampicin eye ointment or $1 \%$ chloramphenicol eye ointment was applied by the patient 3 times daily for a period of $3,4,6$ or 7 weeks.

FOLLOW-UP INVESTIGATIONS

After the initial clinical and laboratory examination treatment was started, and follow-up examinations, with the collection of specimens, were made at weekly intervals for 4 weeks, then at 6 and 8 weeks, and were continued at $3,6,12$, and 18 months after treatment.

\section{Results}

Sixty-three patients were included in this study. The ratio of male to female was $32: 31$. The ages of the patients ranged from 10 days to 49 years, but the majority were between 20 and 40 years old.

The allocations of the 63 patients with various syndromes of infection of the eye by $C$. trachomatis to treatment with either rifampicin or chloramphenicol is shown in Table 2 .

Chloramphenicol was the first drug to be tested in 4- and 6-week courses.

Rifampicin was given first as a 3-week course. The duration of treatment was then increased from 4 to 6 and 7 weeks for several groups of patients.

\section{CHLORAMPHENICOL TREATMENT}

The results of the treatment with chloramphenicol for 4 or 6 weeks are shown in Table 3 and Fig. 1. In general a slight improvement in the clinical signs and symptoms followed the treatment. The average clinical score in patients treated for 6 weeks reduced from 41 to 28 at the end of the treatment. Microbiologically all patients treated for 4 weeks remained positive for $C$. trachomatis. Of those treated for 6 weeks 7 out of 10 were still positive for $C$. trachomatis. The 3 patients with negative culture for C. trachomatis at the end of 6 weeks' treatment were still clinically suffering from a moderate follicular conjunctivitis. Because of the activity of the disease and positive cell culture all patients

Table 3 Number of patients cured following treatment with rifampicin or chloramphenicol eye ointment

\begin{tabular}{llllll}
\hline $\begin{array}{l}\text { Period of } \\
\text { treatment } \\
\text { (weeks) }\end{array}$ & Chloramphenicol & & \multicolumn{2}{l}{ Rifampicin } \\
\cline { 5 - 6 } \cline { 5 - 6 } 3 & & Microbiological & & Clinical & Microbiological \\
\hline 4 & $0 / 7$ & $0 / 7$ & $0 / 3 *$ & $0 / 3$ \\
6 & $0 / 12$ & $3 / 12$ & & $4 / 5$ & $4 / 5$ \\
7 & & & $26 / 29$ & $24 / 29$ \\
& & & $7 / 7$ & $7 / 7$ \\
\hline
\end{tabular}

* Number of patients cured/Number of patients treated.

Table 2 Allocation of patients with various ocular syndromes of infection by Chlamydia trachomatis to treatment with either rifampicin $(R)$ or chloramphenicol $(C)$

\begin{tabular}{|c|c|c|c|c|c|c|c|c|c|c|}
\hline \multirow{2}{*}{$\frac{\text { Syndrome }}{\text { Drug used }}$} & \multicolumn{2}{|c|}{$\begin{array}{l}\text { Ophthalmia } \\
\text { neonatorum }\end{array}$} & \multicolumn{2}{|c|}{$\begin{array}{l}\text { Inclusion } \\
\text { conjunctivitis }\end{array}$} & \multicolumn{2}{|c|}{$\begin{array}{l}\text { TRIC punctate } \\
\text { keratoconjunctivitis }\end{array}$} & \multicolumn{2}{|c|}{ Trachoma } & \multicolumn{2}{|c|}{ Totals } \\
\hline & $\boldsymbol{C}$ & $\boldsymbol{R}$ & $C$ & $\boldsymbol{R}$ & $C$ & $\boldsymbol{R}$ & $C$ & $\boldsymbol{R}$ & $C$ & $\boldsymbol{R}$ \\
\hline \multicolumn{11}{|l|}{ Weeks of treatment } \\
\hline 3 & $\mathbf{0}$ & $\mathbf{0}$ & $\mathbf{0}$ & 3 & $\mathbf{0}$ & 0 & $\mathbf{0}$ & $\mathbf{0}$ & $\mathbf{0}$ & 3 \\
\hline 4 & $\mathbf{0}$ & 1 & 5 & 1 & 2 & 3 & $\mathbf{0}$ & $\mathbf{0}$ & 7 & 5 \\
\hline 6 & $\mathbf{0}$ & 6 & 7 & 16 & 4 & 5 & 1 & 2 & 12 & 29 \\
\hline \multirow[t]{2}{*}{7} & $\mathbf{0}$ & $\mathbf{0}$ & $\mathbf{0}$ & 3 & $\mathbf{0}$ & 4 & 0 & 0 & $\mathbf{0}$ & 7 \\
\hline & & & & & & & & & 19 & 44 \\
\hline
\end{tabular}


Intensity of clinical signs and symptoms
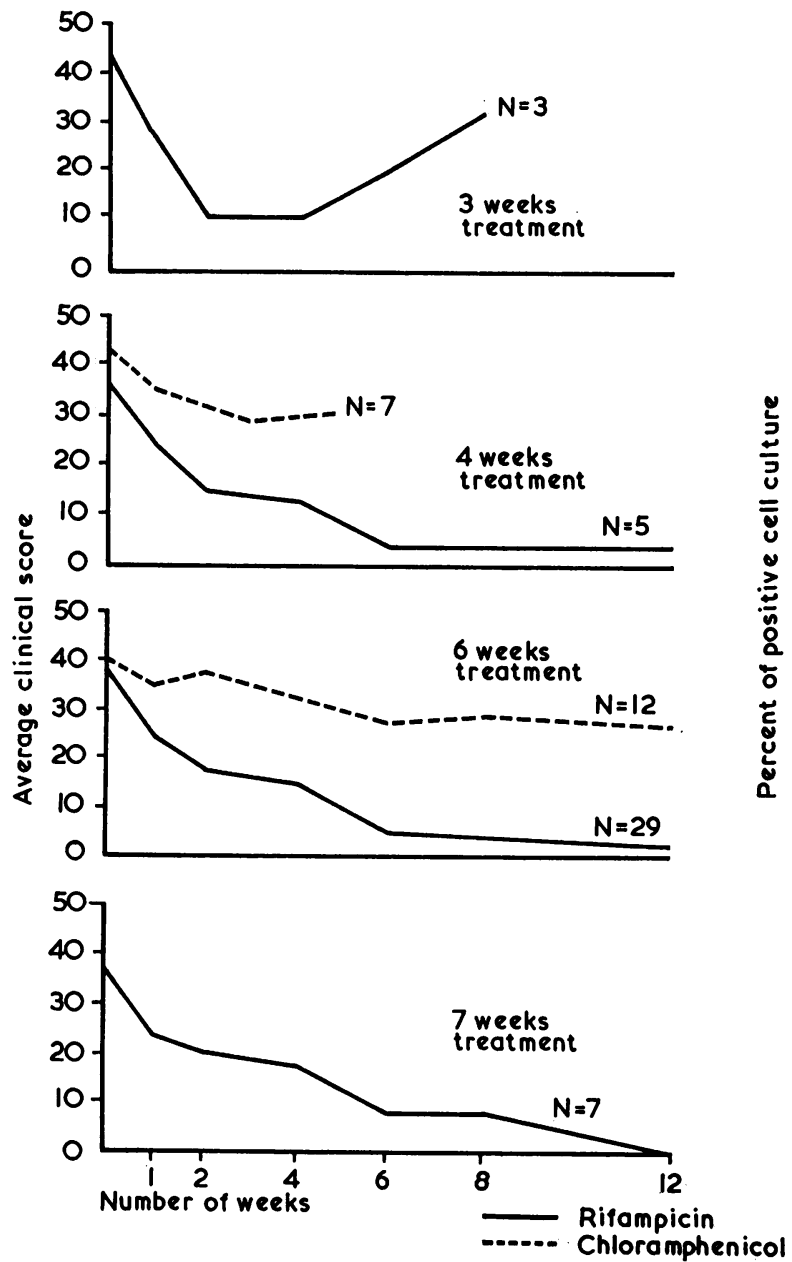

Percent positive cell culture for chlamydia trachomatis
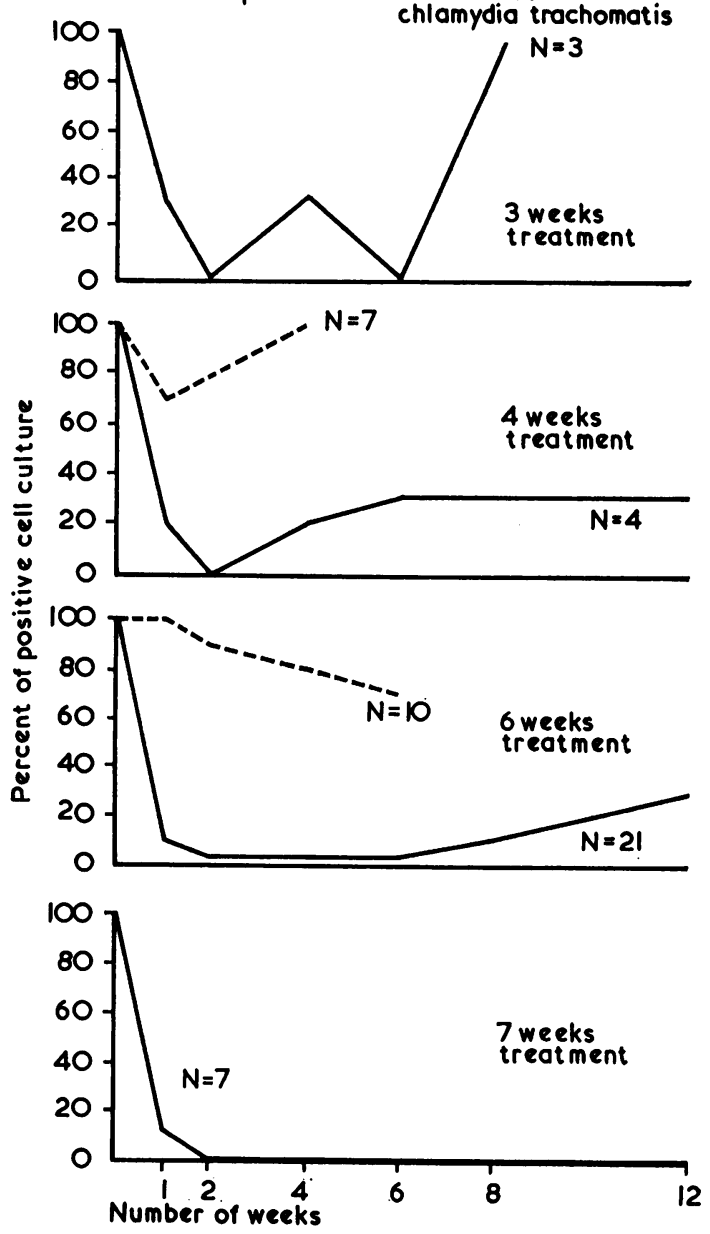

Fig. 1 Changes in the average clinical score and percent positive cell culture after treatment with rifampicin or chloramphenicol

except 1 were treated with rifampicin or tetracycline eye ointments. The 1 patient who partially improved during 6 weeks' treatment with chloramphenicol was followed up for 3 months. No changes were observed in his clinical signs and he remained positive for cell culture for C. trachomatis until he was treated with tetracycline ointment.

\section{RIFAMPICIN TREATMENT}

Three weeks' therapy

The clinical and laboratory findings in 3 patients included in this study are shown in Table 3 and Fig. 1. The average clinical scores before treatment and at 4 weeks after treatment were 43 and 10 respectively, showing a marked improvement in the clinical signs.
Microbiologically only 1 patient was positive for C. trachomatis at 4 weeks' follow-up. One week later this patient showed an active recurrence of infection and was then cured by a further course of this treatment. The remaining 2 patients showed an increase in the signs of activity in their eyes with an average clinical score of 20 at 6 weeks, increasing to 33 at 8 weeks after the treatment. All 3 had again become agent-positive by the eighth week.

Four weeks' therapy

The result of the treatment of 5 patients with rifampicin eye ointment for 4 weeks is shown in Table 3 and Fig. 1. The average clinical score before the treatment was 37 , decreasing to 13 at the end 
of 4 weeks' treatment. The follow-up observations up to 12 weeks showed no clinical activity in 4 patients. The other patient-an adult with the inclusion conjunctivitis syndrome who was microbiologically positive at the end of 4 weeks-showed a slight degree of activity in her clinical signs at 12 weeks. Two weeks later she developed a moderate recurrence of inflammation with a positive cell culture for $C$. trachomatis.

\section{Six weeks' therapy}

The clinical and microbiological findings in 29 patients treated for 6 weeks are shown in Table 3 and Fig. 1. The average clinical scores before and at the end of 6 weeks' treatment were 38 and 5 respectively. The clinical examination of these patients at 12 weeks showed a moderately active disease in only 3 adults, who were originally suffering from inclusion conjunctivitis or TRIC punctate keratoconjunctivitis. The cell culture was positive for C. trachomatis in these 3 patients. Two of these received further treatment with tetracycline eye ointment. The third patient, who was followed up for 6 months, showed a moderate follicular conjunctivitis with a positive cell culture during this period. The follow-up investigations in 12 patients over 1 year and 6 patients over 18 months showed no clinical or microbiological signs of infection in their eyes.

Microbiologically at 12 weeks' follow-up 6 out of 21 patients tested were positive for $C$. trachomatis. Of these 6 patients only 3 developed a recurrence of inflammation. The cell cultures from another patient were positive only once at 12 weeks. The clinical and microbiological findings in this patient at 6 and 12 months' follow-up remained negative. The other 2 patients with positive cell culture at 12 weeks were babies with TRIC ophthalmia neonatorum. Although both babies clinically responded well to the treatment, one remained positive for $C$. trachomatis during the treatment and the second baby became positive at 12 weeks' follow-up. The frequent clinical examinations in these babies over 1 year showed no signs of active disease in their eyes, though 1 baby remained positive for $C$. trachomatis for 3 months and the other one for 6 months.

\section{Seven weeks' therapy}

In 7 patients who were treated for 7 weeks the average clinical scores before and at 8 weeks and 12 weeks after treatment were 37,8 , and 2 respectively (Table 3 and Fig. 1). Microbiologically all the 7 patients were negative for $C$. trachomatis at 8 weeks' follow-up. In 4 of these patients who were followed for over 1 year the clinical and microbiological investigation remained negative.

\section{Adverse reaction}

Three of the 44 patients treated with rifampicin eye ointment developed an adverse reaction 2 to 4 weeks after the start of treatment. This consisted of increased watering soon after application of the ointment, with swelling of the lids together with oedema and increased hyperaemia of the bulbar and palpebral conjunctiva. After treatment was stopped the symptoms and signs subsided in less than 1 week in each case. These patients were removed from the trial. In 4 other patients slight irritation, discomfort, and watering lasting 10 to 50 minutes were reported after each application of the ointment. These patients, however, completed the prescribed course of treatment.

\section{ANTIBIOTIC SENSITIVITY}

With an irradiated McCoy cell-culture the minimum concentration that inhibits the development of C. trachomatis inclusions was $0.05 \mu \mathrm{g} / \mathrm{ml}$ for rifampicin and 1.0 for chloramphenicol against a variety of $C$. trachomatis serotypes. The minimum lethal concentration of rifampicin or chloramphenicol that inhibits the multiplication of $C$. trachomatis after removal of the drug was 1.0 and $5.0 \mu \mathrm{g} / \mathrm{ml}$ respectively.

In 3 patients who were not cured after a 6-week course of treatment with rifampicin $C$. trachomatis isolates recovered from them before and after the treatment were all shown to be inhibited by $0.05 \mu \mathrm{g} /$ $\mathrm{ml}$ of rifampicin. Thus resistance to rifampicin had not developed in these agents after treatment with rifampicin.

\section{Discussion}

In this series of studies we found that after 1 week's treatment with $1 \%$ rifampicin eye ointment used 3 times daily the signs and symptoms were markedly diminished and $87 \%$ of the 44 previously positive patients became negative for $C$. trachomatis.

Although the number of patients included in the 3-week course of treatment was small, it appears that 3 weeks' treatment is not sufficient for the complete eradication of $C$. trachomatis. Of the patients treated for either 4 or 6 weeks 1 out of 5 and 3 out of 29, respectively, were not cured. There was an initial clinical response to the treatment in these 4 patients, but the inflammation recurred 8 to 12 weeks later. The other 2 patients (babies with TRIC ophthalmia neonatorum) clinically responded well to the treatment, but microbiologically they remained 
positive for a period of 3 to 6 months. Although there was no obvious default in application of the ointment in these patients, it is possible that these babies may not have received the ointment in their eye according to the instructions.

The long-term follow-up studies (up to 18 months) indicate that thrice daily treatment with $1 \%$ rifampicin eye ointment for a period of 6 to 7 weeks can cure the disease in $90 \%$ or more of patients with ocular paratrachoma in London.

In a placebo-controlled trial in Southern Tunisia Dawson et al. (1975a) have shown that, in the short term, $1 \%$ rifampicin eye ointment applied twice daily for 10 weeks markedly benefited severe hyperendemic trachoma.

Several authors have claimed that chloramphenicol topical therapy is effective against trachoma (Choumakov et al., 1951 ; Leo, 1951 ; Scuderi and Bellomio, 1952; Vasilieva, 1958). In this study we found that a 4- to 6-week course of treatment with $1 \%$ chloramphenicol eye ointment 3 times a day appeared to reduce the severity of clinical signs and symptoms slightly but did not cure the infection nor did it eliminate the $C$. trachomatis. Hence there appears to be no place for chloramphenicol ointment in the treatment of $C$. trachomatis infections

The authors are indebted to colleagues at Moorfields Eye Hospital for referring their patients and to the members of the External Eye Disease Clinic for their collection of clinical specimens and to Lepetit Pharmaceuticals Ltd. for provision of drugs and facilities for laboratory tests.

This work was financially supported by the Locally Organised Clinical Research Grant, Moorfields Eye Hospital, and by a grant from the Department of Health and Social Security.
References

Becker, Y. (1972). Rifampicin-an anti-trachoma antibiotic. Israel Journal of Medical Sciences, 8, 1110-1113.

Becker, Y., Asher, Y., Himmel, N., and Zakay-Rones, Z. (1970). Anti-trachoma activity of rifampicin and rifampicin SV derivatives. Nature, 225, 454-455.

Choumakov, M. P., Fradkin, M. Y., Schlykova, B. D., Avakyan, A. A., and Zaytzeva, M. S. (1951). A new method of treatment of trachoma with chloramycetin and syntomycin. Vestnik Oftalmologie, 30, 3-9.

Darougar, S., and Jones, B. R. (1971). Conjunctival swabbing for the isolation of TRIC agent (Chlamydia). British Journal of Ophthalmology, 55, 585-590.

Darougar, S., Kinnison, J. R., and Jones, B. R. (1970). Simplified irradiated McCoy cell culture for isolation of Chlamydia. In 'Trachoma and related disorders caused by Chlamydial agents' (ed. R. Nichols). Excerpta Medica International Congress Series, 223, 64-70.

Dawson, C. R., Hoshiwara, I., Daghfous, T., Messadi, M., Vastine, D. W., and Schachter, J. (1975a). Topical tetracycline and rifampicin therapy of endemic trachoma in Tunisia. American Journal of Ophthalmology, 79, 803-811.

Dawson, C. R., Jones, B. R., and Darougar, S. (1975b). Blinding and non-blinding trachoma: assessment of intensity of upper tarsal inflammatory disease and disabling lesions. Bulletin of the World Health Organisation, 52, 279-282.

Leo, E. (1951). Risultati della terapia con cloramfenicolo nel tracoma (Results of therapy with chloramphenicol in trachoma). Atti della Societa Oftalmologica Italiana, 12, 34-37.

Scuderi, G., and Bellomio, S. (1952). Aureomicino, cloramfenicolo, terramici na nella terapia del trachoma (ricerche comparative) (Aureomycin, chloramphenicol and terramycin in the treatment of trachoma). Rivista italiana del tracoma e di patologica oculare esotica, 4, 121-140.

Treharne, J. D., Day, J., Yeo, C. K., and Jones, B. R. (1977). The sensitivity of Chlamydiae to chemotherapeutic agents. Presented at the meeting on 'Nongonococcal urethritis and related oculogenital infections', Lake Placid, New York, April 1976.

Vasilieva, A. (1958). Traitment des enfants trachomateux par la syntomycine (Treatment of trachoma in children with syntomycin). Rivista italiana del tracoma e di patologica oculare esotica, 35, 232. 